\title{
Características del Trauma Maxilofacial de Tercio Medio Atendido en Unidad de Emergencia del Complejo Asistencial Barros Luco
}

\author{
Characteristics of Middle Face Trauma treated in an \\ Emergency Unit of the Barros Luco Healthcare Unit
}

\author{
Alfio Secchi A.'; Joaquín Vázquez D. 1; Jorge Moreno V.2; Felipe Astorga M. ${ }^{2,3}$ \& Pablo Amorín T. ${ }^{2}$
}

SECCHI, A. A.; VÁZQUEZ, D. J.; MORENO, V. J.; ASTORGA, M. F. \& AMORÍN, T. P. Características del trauma maxilofacial de tercio medio atendido en unidad de Emergencia del Complejo Asistencial Barros Luco. Int. J. Odontostomat., 15(1):94-101, 2021.

RESUMEN: EI Trauma Maxilofacial (TMF) es una patología que presenta alta frecuencia en la actualidad, constituyendo un problema global de salud para los centros de alta complejidad y los equipos multidisciplinarios involucrados en su manejo y resolución.(neurología, maxilofacial, otorrinolaringología, oftalmología). Su etiología y epidemiología varía significativamente en la literatura. El objetivo de este estudio es caracterizar a los pacientes atendidos en un hospital del sector sur de Santiago, Chile, identificando sus factores sociales y clínicos, clasificando los principales tipos de fractura a nivel de tercio medio facial. Se realizó un estudio observacional retrospectivo con los Datos de Atención de Urgencia (DAU) de los pacientes atendidos en el Servicio Dental de la Unidad de Emergencia del Complejo Asistencial Barros Luco (CABL), analizando el sexo, edad, comuna de procedencia, etiología del trauma, categorización según el "Emergency Severity Index", horario de ingreso y evaluación, estructuras óseas comprometidas de los pacientes con diagnóstico final de Fractura en Tercio Medio Facial. Se utilizaron estimadores puntuales de porcentaje, media y desviación estándar para representar las variables. Se calculó la significancia de relación de variables independientes, con valor de $p<0,05$ estadísticamente significativo por el tamaño muestral. Población principalmente afectada corresponden a hombres versus mujeres (2.4:1), y entre segunda y tercera década de vida. Principal etiología identificada fue accidentes en el hogar $(n=73)$, seguidos de agresiones $(n=55)$. La mayoría de los pacientes ingresan con un riesgo C4 $(n=107)$ y C3 $(n=64)$. Fractura más frecuentemente es la nasal $(46 \%)$, seguidas de complejo orbitario (11.3\%) y arco cigomático (7.9\%). Es necesario mantener actualizada la epidemiología del TMF considerando la complejidad de su manejo temprano, morbilidades al mediano y largo plazo, con el objetivo de tener un rol activo en su prevención y tratamiento.

PALABRAS CLAVE: etiología, fractura, tercio medio, trauma.

\section{INTRODUCCIÓN}

La región y estructuras maxilofaciales están expuestas a un variado tipo de traumatismos de diverso origen, como lo son agresiones, trauma de alta energía o accidentes domésticos, entre otros.

En este escenario el Trauma Maxilofacial (TMF) constituye una preocupación global y constante para el sistema de salud, en especial para los centros hospitalarios de alta complejidad donde son manejados mayoritariamente por equipos multidisciplinarios producto de la complejidad de su tratamiento.

La importancia de este cuadro radica en las potenciales complicaciones asociadas directamente a él, como hemorragias severas, fallas respiratorias, desórdenes funcionales y complicaciones estéticas locales (Yang et al., 2017), con gran importancia a nivel de salud pública, por la morbilidad y mortalidad que con-

\footnotetext{
${ }^{1}$ Cirujano Dentista, Complejo Asistencial Barros Luco, Pasante, Santiago, Chile.

${ }^{2}$ Cirujano Dentista, Complejo Asistencial Barros Luco, Staff, Santiago, Chile.

${ }^{3}$ Cirujano Maxilofacial, Complejo Asistencial Barros Luco, Staff, Santiago, Chile.
} 
SECCHI, A. A.; VÁZQUEZ, D. J.; MORENO, V. J.; ASTORGA, M. F. \& AMORÍN, T. P. Características del trauma maxilofacial de tercio medio atendido en unidad de Emergencia del Complejo Asistencial Barros Luco. Int. J. Odontostomat., 15(1):94-101, 2021.

llevan, así mismo generan un alto costo en términos económicos, con mayor énfasis en países menos desarrollados donde los recursos para su manejo son limitados (Shayyab et al., 2012).

El primer manejo realizado por lo equipos en los servicios de Emergencia en pacientes con TMF y/ o TEC, es el presentado en las guías "Advanced Trauma Life Support" (ATLS) (Rezaei et al., 2017; Abosadegh \& Rahman, 2018). La evaluación clínica del Trauma Maxilofacial debe ser minuciosa y organizada; con respecto al tercio medio es relevante considerar estructuras que se relacionan con la mayor frecuencia, propuesta por autores; signos y síntomas se detallan a modo general en :

- Fracturas nasales: edema fronto-nasal, epistaxis, asimetría pirámide nasal, dolor local, crépito y enfisema. El control es trascendental para descartar hematoma septal (Mardones et al., 2011).

- Fracturas Orbitarias: equimosis periorbitaria, alteración del movimiento ocular (supraducción), enoftalmo, diplopía, hipoestesia de región geniana ipsilateral.

- Fracturas naso-orbito-etmoidal: aplanamiento puente nasal, disminución de proyección de pirámide nasal, equimosis orbitaria bilateral, telecanto traumático.

- Fractura cigomática: edema geniano, equimosis e hipoestesia región geniana, trismus, escalones óseos palpables.

Fracturas extendidas:

- Lefort I: edema en labio superior, equimosis en fondo de vestíbulo y palatino posterior, enfisema en región geniana y cigomática, movimiento en bloque de maxilar superior, mordida abierta anterior.

- Lefort II: crépito, escalones, equimosis bipalpebral, equimosis subconjuntival, epistaxis bilateral, mordida abierta, parestesia geniana

- Lefort III: Se agrega a los mismos de Lefort II el alargamiento de cara (Hernández et al., 2010).

El examen imagenológico es necesario como apoyo diagnóstico, incluyendo radiografías (posteroanterior de cráneo, telerradiografía de perfil, de huesos nasales) y como gold standard la Tomografía Computarizada (TC) (Nastri \& Gurney, 2016; Dreizin et al., 2018). La unidad de emergencia del CABL posee recursos para ambos exámenes.

La cirugía de contención de daños es trascendental en el manejo de urgencia, en este contexto el aseo de heridas debe ser riguroso para realizar eva- luación de extensión, complejidad y relación de estructuras dañadas con la finalidad de realizar un manejo adecuado y posterior síntesis de tejidos en estructuras maxilofaciales, siendo necesario el control de hemorragias así como también la preservación de cobertura para una correcta cicatrización.

El Complejo Asistencial Barros Luco (CABL), centro de atención de alta complejidad ubicado en la ciudad de Santiago, se sitúa como el principal centro de derivación, diagnóstico y tratamiento de población adulta perteneciente al Servicio de Salud Metropolitano Sur (SSMS), manteniendo una población designada y beneficiaria de 1 millón 158 mil personas (10 comunas anexadas), las cuales ingresan al Centro Asistencial por la urgencia de la Unidad de Emergencia del CABL, lugar donde se constatan mayoritariamente los casos de TMF. Dentro de dicha unidad, el registro clínico de los pacientes es realizado en un instrumento denominado "Dato de Atención de Urgencia" (DAU), el cual contiene información de carácter crítico personal de los pacientes, social y clínica, en base a la cual se puede estudiar la prevalencia, manejo y proceso diagnóstico de los diversos cuadros clínicos presentados.

Desde el punto de vista etario en las características estudiadas del TMF, la mayoría de los autores concuerdan que la mayor prevalencia se presenta en pacientes masculinos, entre la tercera y quinta década (Abosadegh \& Rahman).

El presente artículo tiene como objetivo analizar las características del TMF, en específico de tercio medio facial, de los pacientes atendidos en el Servicio Dental de la Unidad de Emergencia del CABL, por medio del análisis de los DAU.

\section{MATERIAL Y MÉTODO}

Se recopiló la información proveniente de los DAU, del periodo comprendido entre enero del 2018 a junio de 2019. Este documento clínico constituye el registro clínico de la atención recibida del paciente, presentando información de carácter crítico (nombres e identificaciones de los pacientes), estadísticos sociales (previsión, comuna de procedencia, medio de transporte al centro), y clínicos (sexo, edad, categorización de riesgo ESI, tipo de accidente, hora de ingreso, examen físico, exámenes complementarios, diagnósticos). 
SECCHI, A. A.; VÁZQUEZ, D. J.; MORENO, V. J.; ASTORGA, M. F. \& AMORÍN, T. P. Características del trauma maxilofacial de tercio medio atendido en unidad de Emergencia del Complejo Asistencial Barros Luco. Int. J. Odontostomat., 15(1):94-101, 2021.

Los datos críticos no fueron entregados al grupo investigador y solo los dos últimos tipos, estadístico social y clínico fueron los utilizados para el estudio.

La información fue ordenada y analizada por los investigadores usando como plataforma Microsoft Excel $^{\mathrm{TM}}$, usando para este estudio los que presentaran como motivo diagnóstico final Fracturas de Tercio medio facial.

Se incluyó al análisis final todo registro que presentara una descripción íntegra del caso clínico, y fueron excluidos todos los registros cuyas variables se encontraran incompletas, y también los registros de pacientes con edad menor a 12 años.

La información recopilada fue organizada de acuerdo con el tipo de variable:

- Sexo

- Edad en rangos etarios por décadas

- Estructuras anatómicas óseas comprometidas en total y por lado (Fractura Orbito Cigomática izquierda (FOCl), Fractura Orbito Cigomática derecha (FOCD), Fractura Nasoetmoidal (FNE), Fractura Seno Maxilar (FSM), Fractura Nasal (FN), Fractura Lefort I (FL I), Lefort II (FL II), Fractura Arco Cigomático (FA), Fractura Maxilo Malar izquierda (FMMI), Fractura Maxilo Malar derecha (FMMD), Fractura Orbitaria derecha (FOD), Fractura Orbitaria izquierda (FOI).

Los patrones de fractura como Lefort III, Panfacial y Alveolar no fueron consignados dentro del estudio por la ausencia de descripciones asociadas a estos patrones en los DAUs entregados (lo que no significa que no se hayan presentado)

- Distribución mensual

- Índice de Gravedad al ingreso (ESI)

- Etiología

- Medio de transporte de ingreso

- Hora de ingreso (AM y PM)

- Comuna de procedencia de acuerdo con su domicilio

Se utilizaron estimadores puntuales de porcentaje (\%), media (md) y desviación estándar (ds) para representar las variables.

Se calculó la significancia de relación de variables independientes con t-test (Pearson y Welch) (realizadas por software SciStat ${ }^{\mathrm{TM}}$ y STATA ${ }^{\mathrm{TM}}$ ) con un valor de $p<0,05$ estadísticamente significativo por el tamaño de la muestra; con Hipótesis nula la variación similar.

\section{RESULTADOS}

De la recopilación, el total de TMF de tercio medio fue de 189 en los 18 meses analizados, es decir, un promedio de 11 por cada mes, equivalente a 2,7 casos por semana. Del total de casos presentados, 133 fueron hombres y 56 mujeres, calculando una razón 2,4:1 respectivamente. Con respecto a la edad se distribuyó de la siguiente manera, 8,46 \% $(n=16)$ entre $12-19$ años, $28,57 \%(n=54)$ entre $20-29$ años, $19,57 \%(n=37)$ entre 30-39 años, 13,7 \% $(n=26)$ en 40-49 años, 8,46 \% ( $n=16)$ entre 50-59 años, 5,82 \% $(n=11)$ entre $60-69$ años, 7,93 \% $(n=15)$ entre $70-79$ años, 4,76 \% ( $n=9)$ entre 80-89 años, 2,64 \% $(n=5)$ entre 90-99 años. Al aplicar t-test de medias entre cada década y el total de frecuencia, obtuvimos un resultado estadísticamente significativo $(p=0,0047)$ interpretando una diferencia de medias de carácter independiente, destacando el alza en frecuencia de pacientes pertenecientes a la década 20-29 años (Tabla I).

El total de fracturas evaluadas en el servicio odontológico de la unidad de emergencia del CABL fue de 227 en 189 pacientes. Estas se clasificaron según lo descrito anteriormente, 7,48 \% $(n=17)$ para FOC izquierda, 4,84\% $(n=11)$ para FOC derecha, 1,32 \% $(n=3)$ para FNE, 11,01 \% $(n=25)$ para FSM, 46,25 \% $(n=105)$ en FN, 3,40 \% $(n=8)$ para LF I, 0,88 \% $(n=2)$ para FL II, 7,92 \% ( $n=18)$ en FAC, 5,72 \% $(n=13)$ en FMM izquierda, 3,08 \% $(n=7)$ para FMM derecha, 7,92 $\%(n=18)$ en FO izquierda, 3,52 \% ( $n=8)$ en FO derecha (Tabla II).

Fue aplicado t-test (según varianza) para analizar relación entre TMFs y género (cada uno por separado), obteniendo un valor estadísticamente no significativo en la diferencia de medias para el género femenino $(p=0,134)$ y para el género masculino $(p=0,536)$, resultado que en su análisis explica que el género masculino y femenino (por separado) no presentaron un carácter independiente al total de fracturas. Al comparar ambos géneros en sus medias se obtuvo un valor estadísticamente no significativo $(p=0,257)$ interpretando que sus medias no presentan un carácter independiente pese a la evidente diferencia en el tamaño muestral de hombres vs mujeres, además existió en ambos sexos una mayor frecuencia de fractura nasal (Fig. 1).

La distribución mensual presentó la siguiente fórmula, 13 pacientes en enero, 7 febrero, 10 en marzo, 8 en abril, 23 en mayo, 6 junio, 22 julio, 20 en agos- 
SECCHI, A. A.; VÁZQUEZ, D. J.; MORENO, V. J.; ASTORGA, M. F. \& AMORÍN, T. P. Características del trauma maxilofacial de tercio medio atendido en unidad de Emergencia del Complejo Asistencial Barros Luco. Int. J. Odontostomat., 15(1):94-101, 2021.

Tabla I. Distribución de pacientes según edad.

\begin{tabular}{lllll}
\hline Edad (años) & Porcentaje (\%) & Media (edad) & Desviación Estándar & Número $(\mathrm{n})$ \\
\hline $12-19$ & 8,46 & 17 & 1,4 & 16 \\
$20-29$ & 28,57 & 25 & 2,6 & 54 \\
$30-39$ & 19,57 & 34 & 2,5 & 37 \\
$40-49$ & 13,7 & 45 & 2,8 & 26 \\
$50-59$ & 8,46 & 55 & 2 & 16 \\
$60-69$ & 5,82 & 65 & 3 & 11 \\
$70-79$ & 7,93 & 75 & 2 & 15 \\
$80-89$ & 4,76 & 85 & 3 & 9 \\
$90-99$ & 2,64 & 93 & 2 & 5 \\
Total & 100 & $\mathbf{4 2}$ & $\mathbf{2 1}$ & $\mathbf{1 8 9}$ \\
\hline
\end{tabular}

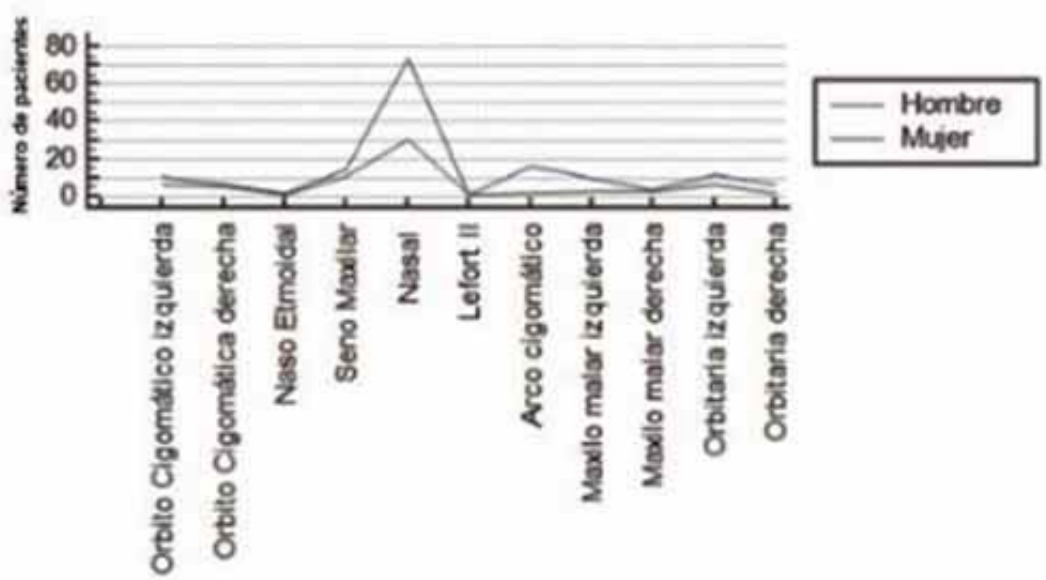

Fig. 1. Frecuencia de patrones de fractura en tercio medio por sexo.

to, 21 en septiembre, 23 en octubre, 16 en noviembre y 20 en diciembre.

Entre los meses de febrero y octubre solo se registraron pacientes masculinos, en contraste con los últimos dos meses que solo fueron pacientes femeninos. Al aplicar t-test para la comparación total de pacientes por mes entre cada sexo, la distribución femenina obtuvo resultado estadísticamente significativo en la diferencias de medias ( $p=0,001$ ) expresando una distribución en alza entre los meses de octubre noviembre y diciembre; en contraste el género masculino presentó resultados estadísticamente no significativos en la diferencia de medias $(p=0,142)$, con distribución heterogénea en cada mes no independiente del total presentado ; de igual manera al comparar ambos sexos se obtuvo un resultado estadísticamente no significativo $(p=0,0638)$, interpretando que las medias de la frecuencia mensual entre cada género presentaron diferencias de carácter no independiente (Fig. 2).
Con respecto a la clasificación de severidad de emergencia al ingreso al servicio según ESI, existió una predominancia de pacientes C3 $(n=107), C 4$ $(n=64), C 2(n=17)$ y finalmente C5 $(n=1)$.

Se puede interpretar al común de pacientes en mediana gravedad y menos grave con la necesidad de esperar la atención de pacientes en riesgo vital, pero con la necesidad de ser evaluados para que su gravedad no aumente (Fig. 3).

Al consignar etiología o el tipo de accidente, este se detalla en el DAU como lo detalla la Tabla III, existiendo mayor frecuencia el registrado como de hogar $(n=73)$, agresión $(n=55)$, otros $(n=43)$, tránsito $(n=12)$ y escolar $(n=6)$ (Tabla III).

En relación con el medio de transporte de ingreso al CABL está fue definida según la ficha DAU en "a pie, ambulancia, vehículos motorizados, vehículos no motorizados, vehículo policial, desconocido". 
SECCHI, A. A.; VÁZQUEZ, D. J.; MORENO, V. J.; ASTORGA, M. F. \& AMORÍN, T. P. Características del trauma maxilofacial de tercio medio atendido en unidad de Emergencia del Complejo Asistencial Barros Luco. Int. J. Odontostomat., 15(1):94-101, 2021.

Tabla II. Distribución de frecuencia de rasgos de fractura según zona anatómica del tercio medio facial afectada y sexo.

\begin{tabular}{lllll}
\hline Ubicación anatómica & Porcentaje (\%) & Número (n) & Mujer & Hombre \\
\hline Orbito cigomática izquierda & 7,48 & 17 & 6 & 11 \\
Orbito cigomática derecha & 4,84 & 11 & 5 & 6 \\
Naso etmoidal & 1,32 & 3 & 1 & 2 \\
Seno maxilar & 11,01 & 25 & 11 & 14 \\
Nasal & 46,25 & 105 & 31 & 74 \\
Lefort II & 0,88 & 2 & 1 & 1 \\
Arco cigomático & 7,92 & 18 & 2 & 16 \\
Máxilo malar izquierda & 5,72 & 13 & 3 & 10 \\
Máxilo malar derecha & 3,08 & 7 & 3 & 4 \\
Orbitaria izquierda & 7,92 & 18 & 6 & 12 \\
Orbitaria derecha & 3,52 & 8 & 2 & 6 \\
Total & $\mathbf{1 0 0}$ & $\mathbf{2 2 7}$ & $\mathbf{7 1}$ & $\mathbf{1 5 6}$ \\
\hline
\end{tabular}

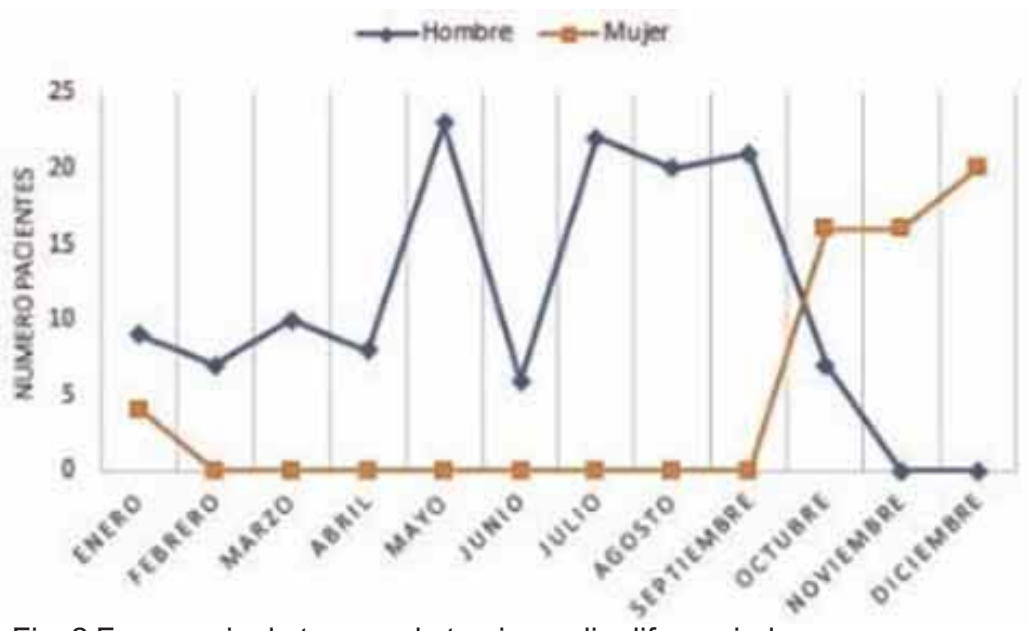

Fig. 2 Frecuencia de trauma de tercio medio diferenciado por sexo y mes.

Tabla III. Distribución del trauma según etiología.

\begin{tabular}{llll}
\hline Tipo de accidente & Hombres & Mujeres & Total \\
\hline Agresión & 34 & 21 & 55 \\
Accidente escolar & 4 & 2 & 6 \\
Hogar & 52 & 21 & 73 \\
Tránsito & 6 & 6 & 12 \\
Otros & 37 & 6 & 43 \\
Total & $\mathbf{1 0 3}$ & $\mathbf{8 6}$ & $\mathbf{1 8 9}$ \\
\hline
\end{tabular}

Tabla IV. Distribución del medio de transporte de ingreso.

\begin{tabular}{llll}
\hline Medio de transporte & Hombres & Mujeres & Totales \\
\hline A pie & 23 & 6 & 29 \\
Transporte motorizado & 54 & 28 & 82 \\
Transporte no & 6 & 6 & 12 \\
Ambulancia & 18 & 7 & 25 \\
Vehículo policial & 1 & 0 & 1 \\
Desconocido & 31 & 9 & 40 \\
Total & $\mathbf{1 3 3}$ & $\mathbf{5 6}$ & $\mathbf{1 8 9}$ \\
\hline
\end{tabular}

El medio de transporte de mayor frecuencia fue el motorizado seguido del consignado como desconocido, interpretando que mayoritariamente el manejo de urgencia prehospitalaria no es realizado por personal capacitado, en relación a esto, el ingreso por ambulancia ocupa el cuarto lugar después del ingreso a pie.

En relación a la hora de ingreso esta se distribuyó en un 31,4\% $(n=60)$ para AM y en $68,6 \%(n=129)$ para PM.

De las comunas asociadas al SSMS, las que presentaron mayor frecuencia en la atención fueron en orden decreciente: San Bernardo $(n=41), \mathrm{Pe}-$ dro Aguirre Cerda $(n=15)$, Lo Espejo ( $n$ = 14), La Cisterna $(n=11)$, San Miguel $(n=7)$, San Joaquín $(n=5)$ y Paine $(n=$ $5)$

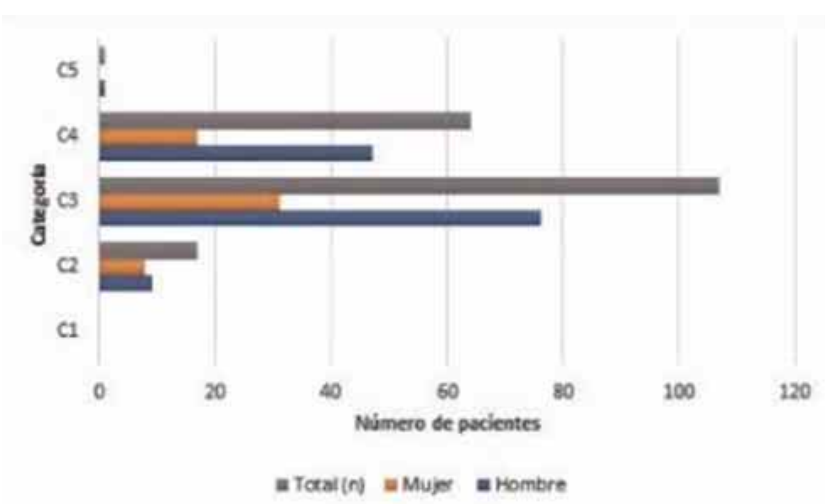

Fig. 3 Número de ingresos de acuerdo con el "Emergency severity index", diferenciado por sexo. 


\section{DISCUSIÓN}

El TMF constituye un problema global de salud debido a la complejidad en su manejo por las estructuras nobles involucradas, su potencial riesgo de vida, morbilidad asociada y costo económico de su tratamiento y posterior rehabilitación; razones que justifican poseer conocimientos en epidemiología local actualizada, con la intención de poseer un rol activo en su prevención y manejo.

A nivel nacional existen diversos artículos acerca de la epidemiología de TMF en servicios de alta complejidad, considerando epidemiología de TMF tratados quirúrgicamente a nivel nacional (Zúñiga et al., 2011; Raposo et al., 2013; Faille Horwood \& Badillo Coloma, 2018; Cuellar et al., 2019) como internacional (Abosadehg et al.; Brasileiro \& Passeri, 2005; Adeyemo et al., 2005; Rezaei et al.). Muchos de ellos entregan un detalle de TMF general y otros aplicados solo a tercio inferior, así como también utilizan muestras de pacientes manejados en evaluación secundaria por el equipo de Cirugía y Traumatología Maxilofacial correspondiente.

Nuestro estudio pone en manifiesto la epidemiología presentada en el servicio de urgencia odontológica de la Unidad de Emergencia del CABL, dentro de la evaluación primaria, siendo información relevante debido a la característica docente asistencial que posee este servicio y la disponibilidad de recursos asociados.

Con respecto a la evidencia existente, las diferencias epidemiológicas en los resultados de dichos artículos la podríamos atribuir a las diversas variables sociales presentes, como la diferente localización geográfica, diferente estrato socioeconómico y nivel cultural.

En relación a la edad más frecuente, al igual que lo propuesto por otros autores (Cuellar et al.; Rezaei et al.; Brasileiro \& Passeri; Zúñiga et al.; Faille Horwood \& Badillo Coloma), la mayor frecuencia de TMF pertenece a hombres entre 20-30 años, con resultado estadísticamente significativo para esta década; la razón hombre:mujer fue de 2,4 / 1, manifestando una persistencia en el sexo masculino.

En la población analizada la fractura más frecuente fue la nasal $(46,5 \%)$, lo sigue, la FO $(11,44$; con predominio en lado izquierdo), seno maxilar (11,2
$\%$, unilateral y/o bilateral) y arco cigomático (7,9 \%). En relación con las fracturas compuestas, la FOC $(15,32 \%)$ y FMM $(8,8 \%)$ presentaron mayor frecuencia, presentado predilección en lado izquierdo, similar a resultados de (Pérez et al.; Faille Horwood \& Badillo Coloma; Abosadegh \& Rahman). La fractura nasal mantiene su alta frecuencia en los TMF al igual que lo expresado por otros autores (Rezai et al.; Gonzalez et al., 2015).

Cabe mencionar que la Unidad de Emergencia del CABL no consta con servicio de otorrinolaringología, razón por la cual el servicio odontológico realiza la evaluación primaria (contención de lesiones) de estos pacientes para su posterior derivación al Servicio de Otorrinolaringología del CABL en un segundo tiempo para su manejo.

Respecto a la clasificación ESI, categorizada al ingreso del paciente a la Unidad de Emergencia; los pacientes expresados en mayor frecuencia en la unidad dental corresponden a los clasificados como C3 y C4, interpretado como pacientes de mediana gravedad y fuera de riesgo vital respectivamente, presentando valores marginales para clasificaciones de mayor riesgo $\mathrm{C} 1$ y $\mathrm{C} 2$, siendo este tipo de pacientes vistos en primera instancia por los equipos de Medicina de Urgencia, solicitando en un segundo tiempo la evaluación por el Servicio Dental, con los pacientes estabilizados.

En base a la distribución mensual, el género masculino no evidenció un resultado estadísticamente significativo, en contraste el género femenino se distribuyó entre los meses de octubre, noviembre diciembre y enero.

Como se ha mencionado anteriormente, el TMF posee variabilidad en su etiología debido a los diversos factores que lo envuelven. En nuestro análisis el accidente en hogar (caídas comunes o de altura) presentó la mayor frecuencia y porcentaje $(n=$ 73 ) $38,6 \%$, seguido de la agresión o violencia $(n=$ 55) $29,1 \%$, datos que se difieren de lo propuesto por autores nacionales de otros centros hospitalarios pertenecientes a otra región o comuna. Faille Horwood \& Badillo Coloma manifiestan las agresiones y accidentes automovilísticos dentro de mayor frecuencia, mientras que González et al. (2015) proponen a los accidentes automovilísticos como la mayor causa de TMF (Faille Horwood \& Badillo Coloma; Gonzalez et al.). Zuñiga y colaboradores realizaron un estudio retrospectivo desde el Servicio 
SECCHI, A. A.; VÁZQUEZ, D. J.; MORENO, V. J.; ASTORGA, M. F. \& AMORÍN, T. P. Características del trauma maxilofacial de tercio medio atendido en unidad de Emergencia del Complejo Asistencial Barros Luco. Int. J. Odontostomat., 15(1):94-101, 2021

de Cirugía Maxilofacial del CABL, pesquisando resultados similares a los nuestros con respecto a la etiología del TMF, siendo caídas comunes y agresiones los más predominantes (Zúñiga et al.).

La hora de ingreso con mayor frecuencia perteneció al rango PM de lo cual no encontramos evidencia sólida que justifique.

\section{CONCLUSIONES}

Creemos necesario mantener actualizada la epidemiología del TMF en los centros hospitalarios de nuestro país, entendiendo que en muchos de ellos, los servicios dentales de las unidades de Urgencia y Emergencia, poseen un rol activo en la evaluación y manejo primario. Muchos de estos centros de alta complejidad poseen carácter docente-asistencial, razón en la que nos sustentamos para remarcar la importancia epidemiológica relacionada a cada zona geográfica, con el objetivo de prevenir y tratar de manera efectiva este tipo de patología y su diversa variedad.

El TMF de tercio medio presentó mayor frecuencia en el sexo masculino específicamente entre la 2da y 3ra década, resultados similares a los propuestos por la evidencia nacional e internacional.

En relación a las estructuras comprometidas, la fractura nasal presentó mayor frecuencia, seguido de fractura orbito cigomática y maxilo malar (con predominio del lado izquierdo), resultados similares a los propuestos en reportes internacionales.

En relación a su distribución mensual, el sexo femenino presentó un reparto estadísticamente significativo (octubre-noviembre-diciembre-enero), consideramos que no representa un realidad constante y es necesario aumentar tamaño muestral para tener un mejor analisis; dentro del DAU no fue posible determinar la presencia de violencia intrafamiliar (VIF) lo cual sería interesante analizar en un próximo estudio.

Con respecto a la categoría ESI, la mayoría del TMF tercio medio se presentó como mediana gravedad, recalcando que de igual manera existieron pacientes graves y en riesgo vital; es importante enfatizar que fracturas extendidas y panfaciales conllevarían mayor gravedad y riesgo vital, factor a considerar en un análisis con mayor tamaño muestral.
En relación a la etiología predominante, los accidentes en el hogar presentaron mayor frecuencia, lo que se podría relacionar con la hora de ingreso a la unidad de Emergencia la cual fue mayoritariamente PM, el medio de transporte de vehículo particular fue el más común. De estos datos podríamos interpretar que el manejo pre-hospitalario de traslado no es realizado por el personal de salud, antecedente relevante a consignar en la anamnesis y evaluación primaria.

De las comunas anexadas al SSMS, San Bernardo presenta la mayor frecuencia lo cual es proporcional a la cantidad de habitantes, siendo esta la más populosa, a su vez es una de las más alejadas geográficamente del la Unidad de Emergencia del $\mathrm{CABL}$, podríamos plantear la interrogante acerca de la disponibilidad de servicios SAPU que realicen evaluación de los pacientes politraumatizados que no requieran manejo de urgencia, con la finalidad de no acudir la unidad de emergencia del CABL.

El servicio dental de la Unidad de Emergencia del CABL atiende un promedio de 26 mil pacientes al año, 2 mil 150 mensuales, de los cuales 11 son por TMF de tercio medio, razón que nos incita a realizar más estudios epidemiológicos para analizar variables claves con la finalidad de aumentar la eficacia y eficiencia en su manejo; creemos pertinente mejorar la ficha electrónica existente como dato DAU con el objetivo de mejorar el flujograma y análisis dentro de la atención.

SECCHI, A. A.; VÁZQUEZ, D. J.; MORENO, V. J.; ASTORGA, M. F. \& AMORÍN, T. P. Characteristics of middle face trauma treated in an emergency unit of the Barros Luco Healthcare Unit. Int. J. Odontostomat., 15(1):94-101, 2021.

ABSTRACT: Maxillofacial Trauma (TMF) is a frequent pathology nowadays and presents a global health problem for specialized trauma centers and the multidisciplinary teams involved in its management and treatment (neurology, maxillofacial, otolaryngology, ophthalmology). The objective of this study is to characterize the patients seen in a hospital in the southern sector of Santiago, Chile, identifying their social and clinical factors, classifying the main types of fracture at the level of the facial third middle. A retrospective observational study was conducted with the Emergency Care Data (DAU) of the patients attended in the Dental Service of the Emergency Unit of the Barros Luco Assistance Complex (CABL), extracting the sex, age, commune of Origin, etiology of the trauma, categorization according to the "Emergency Severity Index" evaluation time and compromised bone structures of patients with diagnosis of Fracture in the Third Facial Mid-Section. Point estimators of percentage, mean 
and standard deviation were used to represent the variables. The significance of the relationship of independent variables was calculated, with a value of $p<0.05$ statistically significant for the sample size. Mainly affected population corresponded to men versus women (2.4: 1), and between the second and third decade of life. Main etiology identified were accidents at home $(n=33)$, followed by assaults $(n=$ $55)$. Most of the patients entered with a risk of C4 $(n=107)$ and C3 $(n=64)$. The most frequent fracture was nasal (46 $\%)$, followed by an orbital complex (11.3\%) and a zygomatic arch $(7.9 \%)$. It is necessary to keep the epidemiology of TMF up to date considering the complexity of its early management and morbidities in the medium and long term.

KEY WORDS: etiology, fracture, midface, trauma.

\section{REFERENCIAS BIBLIOGRÁFICAS}

Abosadegh, M. \& Rahman, S. Epidemiology and incidence of traumatic head injury associated with maxillofacial fractures: A global perspective. J. Int. Oral Health, 10:63-70, 2018.

Adeyemo, W. L.; Ladeinde, A. L.; Ogunlewe, M. O. \& James, O. Trends and characteristics of oral and maxillofacial injuries in Nigeria: a review of the literature. Head Face Med., 1:7, 2005.

Brasileiro, B. F. \& Passeri, L. A. Epidemiological analysis of maxillofacial fractures in Brazil: a 5-year prospective study. Oral Surg. Oral Med. Oral Pathol. Oral Radiol. Endod., 102(1):28-34, 2005.

Cuellar, G. J.; Prats, P. M.; C. Reyes, C. D. \& Sanhueza, O. V. Epidemiología del trauma maxilofacial, tratado quirúrgicamente en el Hospital de Urgencia Asistencia Pública: 3 años de revisión. Rev. Cir., 71(6):530-6, 2019.

Dreizin, D.; Nam, A. J.; Diaconu, S. C.; Bernstein, M. P.; Bodanapally, U. K. \& Munera, F. Multidetector CT of midfacial fractures: classification systems, principles of reduction, and common complications. Radiographics, 38(1):248-74, 2018.

Faille Horwood, A. \& Badillo Coloma, Ó. Caracterización de los casos de fracturas maxilofaciales operados en el Hospital Carlos van Buren, Chile, entre los años 2010-2014. Rev. Esp. Cir. Oral Maxilofac., 40(4):169-75, 2018.

González, E.; Pedemonte, C.; Vargas, I.; Lazo, D.; Pérez, H.; Canales. M. \& Verdugo-Avello, F. Facial fractures in a reference center for Level I Traumas. Descriptive study. Rev. Esp. Cir. Oral Maxilofac., 37(2):65-70 2015.

Hernández, N. R. Manejo del trauma facial: Una guía práctica. Rev. Med. Clin. Las Condes, 21(1):31-9, 2010.

Mardones, M. M.; Fernández, T. M. A.; Bravo, A. R.; Pedemonte, T. C. \& Ulloa, M. C. Traumatología máxilo facial: diagnóstico y tratamiento. Rev. Med. Clin. Las Condes, 22(5):607-16, 2011.

Nastri, A. L. \& Gurney, B. Current concepts in midface fracture management. Curr. Opin. Otolaryngol. Head Neck Surg., 24(4):368-75, 2016.

Raposo, A.; Preisler, G.; Salinas, F.; Muñoz, C. \& Monsalves, M. J. Epidemiología de las fracturas maxilofaciales tratadas quirúrgicamente en Valdivia, Chile: 5 años de revisión. Rev. Esp. Cir. Oral Maxilofac., 35(1):18-22, 2013.

Rezaei, M.; Jamshidi, S.; Jalilian, T. \& Falahi, N. Epidemiology of maxillofacial trauma in a university hospital of Kermanshah, Iran. J. Oral Maxillofac. Surg. Med. Pathol., 29(2):110-5, 2017.

Shayyab, M.; Alsoleihat, F.; Ryalat, S. \& Khraisat, A. S. Trends in the pattern of facial fractures in different countries of the world. Int. J. Morphol., 30(2):745-56, 2012.
Yang, C. S.; Chen, S. C. C.; Yang, Y. C.; Huang, L. C.; Guo, H. R. \& Yang, H. Y. Epidemiology and patterns of facial fractures due to road traffic accidents in Taiwan: A 15-year retrospective study. Traffic Inj. Prev., 18(7):724-9, 2017.

Zúñiga, E.; Yoma, T.; Núñez, C.; Quezada, G. \& Sung, H. H. Epidemiological profile of craniofacial trauma in the Hospital Barros Luco Trudeau, Santiago, Chile. Period 2006-2010. Int. J. Oral Maxillofac. Surg., 40(10):P1112, 2011.

\author{
Direccion para correspondencia: \\ Alfio Secchi Álvarez \\ Cirujano Dentista \\ Complejo Asistencial Barros Luco \\ Santiago \\ CHILE
}

Email: alfio.secchi@gmail.com 